\title{
PREVALENCIA Y CORRELACIÓN ENTRE DIAGNÓSTICO CLÍNICO Y ECOCARDIOGRÁFICO DE CARDIOPATÍAS CONGÉNITAS DURANTE EL PERIODO NEONATAL EN EL HOSPITAL REGIONAL DE ICA, PERÚ
}

\author{
Prevalence and Relationship between clinical and echocardiographic diagnosis congenital heart \\ disease during the neonatal period Regional Hospital lca, Peru
}

Ismael Rolando Gonzáles Tipiana ${ }^{1 a d}$, Aracely Guadalupe Rubianes Huamán ${ }^{2 b c}$, Andrea del Carmen Sobrevilla Huamán ${ }^{3 e}$

${ }^{1}$ Facultad de Medicina, Universidad Nacional "San Luis Gonzaga" de Ica. Ica, Perú. "Institución Educativa "San Luis Gonzaga" de Ica. Ica, Perú. ${ }^{3}$ Centro de Salud, San Clemente Pisco. Ica, Perú.

${ }^{a}$ Médico Pediatra, ${ }^{b}$ Licenciada en Ciencias de la Educación, ${ }^{\circ}$ Magíster en Educación: Mención Administración y Planificación de la Educación Superior, ${ }^{d}$ Doctor en Salud Pública, ${ }^{e}$ Médico Cirujano.

\section{RESUMEN}

Objetivo: Determinar la prevalencia y conocer la correlación entre diagnóstico clínico y ecocardiográfico de las cardiopatías congénitas. Asimismo identificar los hallazgos clínicos, principales complicaciones y cardiopatías congénitas más frecuentes en un hospital de la provincia de lca, Perú. Material y métodos: Estudio descriptivo, transversal y clínico correlacional. Se fueron evaluando y revisando todas las historias clínicas de recién nacidos con diagnóstico clínico, radiológico y ecocardiográfico de cardiopatías congénitas. El estudio incluyo a 18 recién nacidos cardiópatas durante el periodo comprendido entre el 1 de enero al 31 de diciembre del 2011. Resultados: De 2369 recién nacidos en el periodo de estudio 18 presentaron cardiopatías congénitas, siendo la prevalencia de 7,6 por 1000 nacidos vivos. Las cardiopatías acianóticas representaron $83 \%$ y las cianotizantes $17 \%$, neonatos a término fueron $77,8 \%$ y 22,2 prematuros. El $33,3 \%$ presentaron bajo al nacer $<2500$ gramos y $66,7 \%$ peso superior a 2500 gramos. El diagnóstico se hizo, durante la primera semana de vida en $77,8 \%$ y 22,2\% después de los 7 días. Las cardiopatías más frecuentes fueron: comunicación interventricular (CIV) 44\%, ductus arterioso persistente y comunicación interauricular (PDA + CIA) 22\%, Comunicación interventricular y Comunicación interauricular (CIV + CIA) 17\%, Anomalía de Ebstein 5,7\%, drenaje venoso pulmonar anómalo total (DVPAT) 5,7\% y transposición de grandes vasos (TGV) 5,7\%. El índice cardiotorácico > 0,6 estuvo presente en el 61\% y 39\% no presentaron alteración. Los hallazgos clínicos encontrados con mayor frecuencia fueron: soplo cardiaco $94 \%$, taquipnea $61 \%$, hepatomegalia $44 \%$ y lactancia entrecortada $22 \%$. La correlación entre diagnóstico clínico y ecocardiográfico de las cardiopatías congénitas correspondió al $77,8 \%$. Siendo las principales complicaciones: insuficiencia cardiaca $56 \%$, infecciones $28 \%$ y $16 \%$ hipertensión arterial persistente. Conclusiones: La prevalencia de cardiopatías congénitas fue 7,6 por 1000 nacidos vivos. Las más frecuentes fueron las acianoticas: comunicación interventricular, comunicación interauricular y ductus arterioso persistente. Los hallazgos clínicos encontrados: soplo cardiaco y taquipnea. La complicación más común fue la insuficiencia cardiaca. Asimismo se demostró una alta correlación entre diagnóstico clínico y ecocardiográfico.

Palabras claves: Cardiopatía congénitas, ecocardiografía.

\section{SUMMARY}

Objective: To determine the prevalence and know the correlation between clinical and echocardiographic diagnosis of congenital heart disease. Also identify the clinical, major complications and congenital heart disease more common in a hospital in the province of Ica, Perú. Methods: Descriptive, transversal and clinical correlation. They were evaluating and reviewing all the medical records of infants with clinical, radiological and echocardiographic congenital heart 
disease. The study included 18 newborns with heart disease during the period from January 1 to December 31, 2011. Results: of 2369 newborns in the study period, 18 had congenital heart disease, with a prevalence of 7, 6 per 1000 live births. A cyanotic heart disease accounted for $83 \%$ and cyanotic $17 \%$, term infants were $77.8 \%$ and $22.2 \%$ premature. The $33.3 \%$ had low birth $<2500$ grams and $66.7 \%$ weight greater than 2500 grams. The diagnosis was made during the first week of life in $77.8 \%$ and $22.2 \%$ after 7 days. The most frequent diseases were: ventricular septal defect (VSD) 44\%, patent ductus arteriosus and atrial septal defect (PDA + CIA) 22\%, interventricular septal and Communication (CIA + CIV) $17 \%$, Ebstein anomaly 5.7\%, total anomalous pulmonary venous drainage (TAPVR) $5.7 \%$ and transposition of the great arteries (TGA) $5.7 \%$. The cardiothoracic ratio $>0.6$ was present in $61 \%$ and $39 \%$ had no alteration. The clinical findings most frequently found were: heart murmur $94 \%$, tachypnea, $61 \%$, hepatomegaly $44 \%$ and $22 \%$ ragged lactation. The correlation between clinical and echocardiographic diagnosis of congenital heart diseases corresponded to $77.8 \%$. It is the major complications: $56 \%$ heart failure, infections $28 \%$ and $16 \%$ persistent hypertension. Conclusions: Prevalence of congenital heart defects was 7.6 per 1000 live births. The most frequent were acyanotic: interventricular communication, atrial septal defect and patent ductus arteriosus and clinical findings: cardiac murmur and tachypnea. The most common complication was heart failure. A high correlation between clinical and echocardiographic diagnosis was also demonstrated.

Keywords: congenital heart disease, echocardiography.

\section{INTRODUCCIÓN}

Las cardiopatías congénitas, con una prevalencia de 4 a 8 de cada 1000 recién nacidos vivos, son los defectos congénitos graves más frecuentes y una de las tres principales causas de mortalidad prenatal y perinatal. Desde hace tiempo se reconocen algunos factores de riesgo asociados, como el antecedente de cardiopatía en alguno de los padres 0 hermanos, enfermedades maternas (alteraciones del tejido conectivo, diabetes, ingestión de ciertos fármacos 0 infecciones), síndromes específicos como el de Down o el de Turner, presencia de malformaciones extracardíacas, retraso del crecimiento intrauterino, polihidramnios $u$ oligohidramnios o la detección de arritmias fetales (bradiarritmias o taquiarritmias) $(1,2$, $3)$.

Los factores medioambientales dan cuenta del $2 \%$ de las cardiopatías congénitas, son modificables por lo tanto en este nivel se puede hacer prevención. Por ejemplo la rubeola, causante del ductus arterioso persistente, radiaciones e ingesta de alcohol relacionados con la comunicación interventricular, la edad materna y el uso de drogas como el litio, trimetadona, insulina y otras son altamente sospechosas. Finalmente en la etiología multifactorial concurren cerca del $90 \%$ de las cardiopatías congénitas, lo que significa que en ciertos individuos, se requieren factores ambientales múltiples para que su enfermedad tenga una expresión clínica. El aumento de la translucidez nucal, evaluable mediante la ecografía entre las semanas 11 y 14 de embarazo, ha sido mencionado como un marcador de riesgo independiente $(4,5)$. Sin embargo, más del $80 \%$ ocurren en embarazos sin factores de riesgo reconocidos hasta el momento $(5,6)$. Varios estudios han demostrado que el diagnóstico prenatal de las cardiopatías, mediante la ecocardiografía fetal puede afectar significativamente la morbimortalidad perinatal, al permitir planear el parto en instituciones con unidades cardiovasculares pediátricas, realizando de esta forma intervenciones terapéuticas inmediatas esenciales para la supervivencia en el período neonatal en el caso de cardiopatías graves $(5,6)$. Es importante diagnosticarlos durante el primer año de vida y actualmente se puede salvar con tratamiento quirúrgico $85 \%$ de estos niños, sea con cirugía paliativa - correctiva. Las cardiopatías con 
cortocircuito de izquierda a derecha constituyen las más frecuentes (Comunicación interventricular, comunicación interauricular y ductus arterioso persistente), seguidas por las patologías estenoticas (estenosis pulmonar y estenosis aortica). A continuación vienen las cardiopatías cianóticas: Transposición de grandes vasos, Tetralogia de Fallot y Drenaje Venoso Pulmonar Anómalo (1, 3, 7). El diagnóstico hasta en un $90 \%$ de los casos se puede hacer con los métodos clínicos tradicionales, vale decir con la auscultación, la radiología y el electrocardiograma (2). El pediatra debe dominar el método de pensamiento científico ordenado que le permita, valiéndose de sus ojos, oídos, una telerradiografía de corazón y grandes vasos y un electrocardiograma orientarse hacia el diagnóstico de las cardiopatías más comunes por ejemplo: un niño acianótico con hiperemia en la radiografía de tórax, tendrá un cortocircuito de izquierda a derecha y por lo tanto será una comunicación interventricular, comunicación interauricular y/o ductus arterioso persistente. En cambio si no tuviera hiperemia, seria algún tipo de estenosis. Si es un niño cianótico sin hiperemia pulmonar, probablemente corresponde a una Tetralogía de Fallot o una atresia tricuspidea. Pero si tuviera hiperemia nos encontraríamos frente a una cardiopatía cianótica grave como la Transposición de grandes vasos u otra $(2,8)$. El objetivo principal de este estudio es determinar la prevalencia y demostrar la correlación entre el diagnóstico clínico y ecocardiográfico de las cardiopatías congénitas en el Hospital Regional de Ica.

\section{MATERIAL Y METODOS}

Se realizó una investigación de tipo descriptiva, transversal y clínico correlacional del comportamiento de las malformaciones cardiacas congénitas en el Servicio Neonatología del Hospital Regional de IcaPerú, durante el período comprendido entre el $1^{\circ}$ de Enero al 31 de Diciembre de 2011. Durante este periodo nacieron 2369, de los cuales 18 presentaron cardiopatías congénitas, siendo la prevalencia de 7,6 por 1000 nacidos vivos. Los datos fueron obtenidos de las historias de los recién nacidos con diagnóstico clínico, radiológico y ecocardiográfico de cardiopatía congénita. Se consignaron la edad gestacional y peso de los neonatos, asimismo si el diagnóstico se realizó durante la primera semana de vida o fue posterior a los 7 días posnatales, también el índice cardiotorácico. Se utilizó una ficha de recolección de datos elaborada por el investigador y validada en el Hospital Regional de Ica. Asimismo los datos se procesaron estadísticamente usando el programa Epilnfo y se realizó un análisis univariado y bivariado con IC $95 \%$ considerando un $p<0,05$ como estadísticamente significativo. Finalmente se confeccionaron tablas y gráficos, para facilitar el análisis y discusión de los resultados. Nuestros objetivos fueron; determinar la prevalencia y conocer la correlación entre el diagnóstico clínico y ecocardiográfico de las cardiopatías congénitas. Asimismo identificar los principales hallazgos clínicos, complicaciones y cardiopatías congénitas más frecuentes.

\section{RESULTADOS}

La prevalencia de cardiopatías congénitas fue de 7,6 por cada 1000 nacidos vivos. Durante este periodo de estudio nacieron 2369. Solamente 18 neonatos presentaron características clínicas, hallazgos radiológicos y estudios de ecocardiografía compatibles con el diagnóstico de cardiopatía congénita. Las cardiopatías acianóticas representaron $83 \%$ y las cianotizantes $17 \%$. Siendo las más frecuentes: comunicación interventricular $44 \%$, ductus arterioso persistente y comunicación interauricular 22\%, comunicación interventricular y comunicación interauricular 17\%, anomalía de Ebstein $5,7 \%$, drenaje venoso pulmonar anómalo total $5,7 \%$ y transposición de grandes vasos $5,7 \%$. (Tab. $\mathrm{N}^{\circ}$ 1) Las características clínicas encontrados con mayor frecuencia fueron: 
soplo cardiaco 94\%, taquipnea $61 \%$, hepatomegalia $44 \%$ y lactancia entrecortada $22 \%$. Recién nacidos a término fueron $77,8 \%$ y $22,2 \%$ prematuros. El $33.3 \%$ presentaron bajo al nacer < 2500 gramos y $66,7 \%$ peso superior a 2500 gramos. El diagnóstico se efectuó, durante la primera semana de vida en $77,8 \%$ y $22,2 \%$ después de los 7 días. El índice cardiotorácico $>0,6$ estuvo presente en el $61 \%$ y $39 \%$ no presentaron alteración. La correlación entre diagnóstico clínico y ecocardiográfico de las cardiopatías congénitas correspondió al 77,8\% (Tabla № 2) Las principales complicaciones fueron; insuficiencia cardiaca $55 \%$, infecciones $28 \%$ y $16 \%$ hipertensión pulmonar persistente (Tabla $\mathrm{N}^{\circ} 3$ )

Tabla № 1 Principales cardiopatías congénitas en el Hospital Regional de Ica. 2011

\begin{tabular}{lccc}
\hline \multicolumn{1}{c}{ VARIABLES } & $\mathbf{n = ~ 1 8}$ & $\%$ & $\mathbf{P}$ \\
\hline Cardiopatías & 3 & 17 & 0,33 \\
Cianótica & & & \\
Anomalía de Ebstein & 1 & 5,6 & \\
DVPAT & 1 & 5,7 & \\
TGA & 1 & 5,7 & \\
Cardiopatías Acianoticas & 15 & 83 & 0,67 \\
CIV & & & \\
PDA + CIA & 8 & 44 & \\
CIV + CIA & 4 & 22 & \\
& 3 & 17 & \\
\hline
\end{tabular}

Tabla $N^{\circ}$ 2: Principales características clínicas, hallazgos radiológicos y ecocardiográficos en recién nacidos con cardiopatías congénitas en el hospital Regional de Ica. 2011

\begin{tabular}{lccc}
\hline \multicolumn{1}{c}{ VARIABLES } & $\mathbf{n = ~} \mathbf{1 8}$ & $\%$ & $\mathbf{P}$ \\
\hline C. Clínicas & 17 & 94 & 0,91 \\
Soplo & 11 & 61 & 0,61 \\
Taquipnea & 8 & 44 & 0,44 \\
Hepatomegalia & 4 & 22 & 0,21 \\
Lactancia entrecortada & & & \\
E. Gestacional & & & \\
$\geq 37$ semanas & 14 & 77,8 & 0,76 \\
< 37 semanas & 4 & 22,2 & 0,22 \\
Peso & & & \\
Mayor o igual a 2,500 grs & 12 & 66,7 & 0,65 \\
Menor a 2,500 grs & 6 & 33,3 & 0,34 \\
Diagnostico & & & \\
Primeros 7 días & 12 & 77,8 & 0,78 \\
$\begin{array}{l}\text { Después de los 7 días } \\
\text { Índice cardiotorácico }\end{array}$ & 4 & 22,2 & 0,22 \\
$\begin{array}{l}\text { Anormal } \\
\text { Normal } \\
\text { Diagnóstico clínico y }\end{array}$ & 11 & 61 & 0,60 \\
Ecocardiografía & 7 & 39 & 0,40 \\
\hline
\end{tabular}

Tabla № 3: Principales complicaciones en recién nacidos con cardiopatía congénita en el Hospital Regional de Ica. 2011

\begin{tabular}{lllcc}
\hline \multicolumn{1}{c}{ VARIABLES } & $\mathbf{n = 1 8}$ & $\%$ & $\mathbf{P}$ \\
\hline Insuficiencia Cardiaca & 10 & 55 & 0,53 \\
Infecciones & 5 & 28 & 0,29 \\
Hipertensión Pulmonar & 3 & 17 & 0,19 \\
\hline
\end{tabular}

\section{DISCUSIÓN}

La prevalencia de cardiopatías congénitas fue de 7,6 por cada 1000 nacidos vivos. Las cardiopatías acianóticas representaron $83 \%$ (15 neonatos) y las cianotizantes 17\% (3 neonatos). Siendo las más frecuentes: comunicación interventricular 44\% (8 neonatos), ductus arterioso persistente $y$ comunicación interauricular 22\%, comunicación interventricular y comunicación interauricular 17\%, anomalía de Ebstein $5,7 \%$, drenaje venoso pulmonar anómalo total $5,7 \%$ y transposición de grandes vasos $5,7 \%$. En Costa Rica en el Hospital Nacional de Niños durante 2006-2007, se encontró una prevalencia de 0,6\% (IC: 95\%: 0,5-0,7). Las cardiopatías congénitas más frecuentes fueron los defectos del tabique interventricular e interauricular, persistencia del conducto arterioso, estenosis valvular pulmonar, defectos del tabique aurículo ventricular, coartación de aorta y tetralogía de Fallot (8). En Noruega la prevalencia global de cardiopatías congénitas fue 12,34 por 1000 nacidos vivos en el periodo comprendido desde 1994 al 2005. La prevalencia por 1000 nacimientos fue el siguiente: defectos conotruncales 1,16, defectos del tabique ventricular 6,65, ductus arterioso persistente, 2,46 y el retorno venoso pulmonar anómalo, 0,11 (9). En la Unidad de Maternidad del Hospital Clínicas de la Universidad Federal de Minas Gerais, Brasil, durante el período, agosto de 1990 y diciembre de 2003, se encontró una prevalencia de 9,58/1000 nacidos vivos. Asimismo se encontró asociación entre el peso al nacer $\leq 2500$ gramos y la edad materna mayor de 35 años (10). En nuestro estudio 33,3\% (6 neonatos) presentaron bajo al nacer $<2500$ gramos $y$ 
$66,7 \%$ (12 neonatos) peso superior a 2500 gramos. En la investigación realizada por la Escuela de Salud Pública de la Universidad de Emory, Atlanta (Georgia) durante 19982005, se logró determinar una prevalencia general de 8,14/1000 nacimientos. Las cardiopatías congénitas más comunes fueron: comunicación interventricular 3,81, comunicación interauricular 1,03, tetralogía de Fallot 0,47 y la transposición de las grandes vasos 0,23 por 1000 nacidos vivos (11). En Asturias (España) durante los años 1990 a 2004, la prevalencia total media de malformaciones cardiacas congenitas fue de 7,52 por 1000 nacimientos. Los defectos cardiacos más frecuentes fueron: comunicación interventricular (2,88 por cada 1000 nacidos vivos), comunicación interauricular (1,03 por cada 1000 nacidos vivos) y la persistencia del ductus arterioso (0,60 por cada 1000 nacidos vivos) (12).

Las características clínicas encontrados con mayor frecuencia fueron: soplo cardiaco 94\% (17 neonatos), taquipnea $61 \%$ (11 neonatos), hepatomegalia $44 \%$ (8 neonatos) y lactancia entrecortada $22 \%$ (4 neonatos). $77,8 \%$ (14 neonatos) fueron a término y $22,2 \%$ (4 neonatos) prematuros. La correlación entre diagnóstico clínico y ecocardiográfico de las cardiopatías congénitas correspondió al $77,8 \%$. El diagnóstico se hizo, durante la primera semana de vida en $77,8 \%$ (12 neonatos) y $22,2 \%$ (4 neonatos) después de los 7 días. El índice cardiotorácico > 0,6 estuvo presente en el $61 \%$ (11 neonatos) y 39 $\%$ (7 neonatos) no presentaron alteración.

En conclusión la Prevalencia de cardiopatías congénitas fue 7.6 por 1000 nacidos vivos. Las más frecuentes fueron las acianoticas: comunicación interventricular, comunicación interauricular y ductus arterioso persistente. Los principales hallazgos clínicos encontrados: soplo cardiaco y taquipnea. La complicación más común fue la insuficiencia cardiaca. Asimismo se demostró una alta correlación entre diagnóstico clínico y ecocardiográfico.

\section{Correspondencia:}

Ismael Rolando Gonzáles Tipiana

Correo electrónico:

\section{REFERENCIAS BIBLIOGRAFICAS}

1. Gladman G, Silverman E, Yuk L, Luy L, Boutin C, Laskin C, Smallhorn J. Fetal echocardiographic screening of pregnancies of mothers with anti-Ro and/or anti-La antibodies. Am J Perinatol 2002; 19(2):73-80

2. Allan L, Crawford D, Chita S, Anderson $\mathbf{R}$, Tynan $\mathbf{M}$. The familial recurrence of congenital heart disease in a prospective series of mothers referred for fetal echocardiography. Am J Cardiol 1986; 58:334-7

3. Kleinman C, Hobbins J, Jaffe C, Talner N. Echocardiographic studies of the human fetus: prenatal diagnosis of congenital heart disease and cardiac diysrhythmias. Pediatrics 1980; 65:105967

4. Stumpften I, Stumpften A. Effect of detailed fetal echocardiography as part of routine prenatal ultrasonographic screening on detection of congenital heart disease. Lancet 1996; 348:854-7

5. Shinebourne E, Carvalho J. Ethics of fetal echocardiography. Editorial. Cardiol Young 1996; 6:261-3

6. Eapen R, Rowland D, Franklin W. Effect of prenatal diagnosis of critical left heart obstruction on perinatal morbidity and mortality. Am J Perinatol 1998, 4:237- 42

7. Chitty L, Hunt G, Moore J, Lobb M. Effectiveness of routine ultrasonography in detecting fetal structural abnormalities in a low risk population. $\mathrm{Br}$ Med $\mathrm{J} 1991$; 303:1165-0

8. Benavides A, Faerron J, Umaña L, Romero J. Epidemiología y registro de las cardiopatías congénitas en Costa Rica. Rev. Panam Salud Pública. 2011; $30(1): 31-8$ 
9. Leirgul E. y Col. Birth prevalence of congenital heart defects in Norway nationwide study. Bergen and Oslo, Norway. 1994-2009

10. Amorim L, Pires C, Lana A, Campos A, Aguiar $\mathrm{R}$, Tibúrcio $\mathrm{J}$, et al. Presentation of congenital heart disease diagnosed at birth: analysis of 29,770 newborn infants. $\mathrm{J}$ Pediatr. 2008;84(1):83-90

11. Mark D. Reller, MD, Matthew J. Strickland, PhD, Tiffany RiehleColarusso MD, William T. Mahle, MD, and Adolfo Correa, MD, PhD Prevalence of Congenital Heart Defects in Metropolitan Atlanta.1998-2005. J Pediatr. 2008 December; 153(6): 807813
12. Rodríguez C. y Col. Epidemiología de las cardiopatías congénitas en Asturias. An Pediatr dic. 2009 (Barc); 71(6): 502509

Recibido: 15/08/2015

Aprobado para publicación: 20/11/2015 